\title{
Analysis of Phase Change Flow and Network Performance on Complex Network Model
}

\author{
Liping Zhang \\ Sichuan Information Technical College, China
}

55217096@qq.com

Keywords: Complex network, phase change flow, network index, data simulation

\begin{abstract}
In this paper, we discuss the phase change flow of network and the change law of the network performance under different traffic input rates. Based on the stability conditions of the and the system, the approximate expressions of the phase change flow of the network traffic are We also derive the analytical results of the queue length, average delay, network throughput and performance indicators and the deviation degree of the network behavior on the stability of the network behavior. At the same time, we carry out the simulation of the data according to the actual situation, and the simulation results show that (1) The global behavior of heterogeneous network heterogeneous topology has local characteristics from the free flow regime to the congestion state. It is a feasible method to improve the network performance by transforming the network topology improve the value of the network traffic flow. (3) As the network load increases, the global behavior of the network is directly from the free flow state to the global congestion state. There is no state between the two states. (4) When the flow rate exceeds the global phase transition, the queue length and the average delay of the packet are increased dramatically, and the maximum value of network throughput is quickly reduced to zero.
\end{abstract}

\section{Introduction}

Phase change flow is an upper bound on the maximum load of a network system under stable conditions, and it is an important global index of network performance analysis and evaluation. make the network have a high phase change flow is often the optimization goal of the network planning problem [1]. In the process of carrying a variety of communication services, the most ideal situation of the network is to maintain the system to the maximum capacity to transmit packets and not to make the system congestion occurs such a critical state. However, in the vicinity of the phase transition flow value, the network often has a wealth of critical behavior characteristics, and its complexity, uncertainty and uncontrollable increase [2]. Therefore, the description of the network behavior near the phase transition flow and the phase change flow value has important guiding significance for the actual network management, such as the early warning and avoidance of congestion, the optimization of network resources and service quality control.

In this paper, we toke the local stability condition of the network node and the global stability condition as the foundation, and proposed an approximate representation method of phase change flow in complex network model $[3,4]$. On this basis, we further analyze the variation law of the network system performance index, such as queue length and delay time under different network load and discuss the key factors that affect the overall behavior of the network and the performance of the network.

\section{Analysis of phase change flow and network performance index}

This section will describe the approximate expression of the phase change flow and the analysis of the behavior of the relevant performance indicators in the vicinity of the phase change flow, and finally data simulation is carried out in this section.

\subsection{Approximate representation of phase change flow}

In complex network theory, the number of nodes reflects the importance of the evolution of the node in the network, and its definition is as follows. 
Assume that the shortest path between the source node $i$ and destination node $j$ is $g(i, j)$, and there are $\mathrm{g}_{\mathrm{y}}(\mathrm{i}, \mathrm{j})$ path through the node $\mathrm{y}$. We define the ratio as:

$$
B_{y}(i, j)=\frac{g_{y}(i, j)}{g(i, j)}
$$

$B_{y}$ describes the importance of $y$ in the communication of the node $i$ and node $j$. We allow the $B_{y}$ to meet the following conditions:

$$
B_{y}=\sum_{i, j \in V} B_{y}(i, i)=\sum_{i, j \in \in} \frac{g_{y}(i, i)}{g_{y}(i, i)}
$$

$\mathrm{B}_{\mathrm{y}}$ is called the betweenness of node $\mathrm{y}$, and it represents the number of nodes in the shortest path in the network that passes through the node y [5].

The betweenness of nodes is a global index of the network. It reflects the role and influence of the node in the whole network. The value of a node is greater, then it shows that the amount of information in the communication process through the node will be more, and it also has great influence on network performance, at the same time the node is more prone to congestion.

Because $\mathrm{M}(\mathrm{M}-1) \bar{\zeta}$ is the normalized coefficient of the betweenness of nodes. Therefore, $\mathrm{M}(\mathrm{M}-1) \bar{\zeta}$ can be used to do normalization processing of $\mathrm{B}_{\mathrm{y}}$, and the results can be expressed by $\varpi_{y}$, that is:

$$
\varpi_{y}=\frac{B_{y}}{M(M-1) \bar{\zeta}}
$$

We call $\varpi_{y}$ as the normalized number of node y. $\varpi_{y}$ represents the ratio of the shortest path to the number of the shortest path through the node $\mathrm{y}$ on the entire network. Like $\mathrm{B}$, it also describes the importance of node y throughout the network.

Assumptions the flow is known on the network, then the flow rate of the node y can be expressed as the product of the total flow and the normalized medium number. For any node $y$ on a uniform network, it is clear that the value of each node is equal, so the value of $y$ can be expressed as:

$$
B_{y}=(M-1) \bar{\zeta}
$$

Here we use the node number to give an approximate representation of the local flow $\lambda_{c}^{\text {loc }}$. We consider the case when the network is in a state of free flow. In each unit time, the total flow generated by the network is $p m \lambda, \mathrm{y}$ is assumed to be an arbitrary node in the network, and the normalized number is $\varpi_{y}$. Therefore, when the network is in the free flow regime, it is known that the flow rate $\mathrm{N}_{\mathrm{y}}(\mathrm{t})$ through the node $\mathrm{y}$ in a unit time can be expressed as $p m \lambda \varpi$. So there are the following conclusions set up.

(5) ${ }^{p m \lambda \sigma_{y} \leq \frac{1}{\varepsilon f}}$

From the above type, we can know that each node can be in a steady state, the rate of input flow rate $\lambda$ should meet the following conditions:

$$
\lambda \leq \min _{y=v}\left\{\frac{1}{p M \bar{\varepsilon} f}\right\}=\min _{y=1}\left\{\frac{1}{p M \bar{\varepsilon} f \cdot \max \left(\sigma_{y}\right)}\right\}
$$

Under this condition, each node is in a steady state, and it is able to transmit the incoming packets in time. The whole system is in the free flow phase. The time experienced by the packet in the network is only the forwarding delay. So we can get the approximate expression of the local flow $\lambda_{c}^{\text {loc }}$ :

$$
\lambda_{c}^{\text {loc }}=\frac{1}{\mathrm{pM} \bar{\zeta} \varpi_{\max }}
$$

Among them, $\varpi_{\max }=\max _{y \in V}\left\{\varpi_{y}\right\}$ is the largest network in the normalized number of nodes. In 
addition, we can get another equivalent form of $\lambda_{c}^{\text {loc }}: \lambda_{c}^{\text {loc }}=\frac{M-1}{\mathrm{p} B_{\max }}$

\subsection{Analysis of performance index behavior near the phase change flow}

In addition to knowing the phase change traffic of the network, we should also care about the behavior characteristics of the network system under different loads. Only by understanding the queue length and the average delay of the nodes in the steady state, it is possible for us to implement the appropriate buffer strategy to gain more feedback time for the congestion control mechanism [6, 7].

\subsubsection{Packet queue behavior analysis}

(1) When $0<\lambda<\lambda_{c}^{\text {loc }}$

At this time, the network is in the free flow state, the average delay of packets only contains the average forwarding time, while the average waiting time is zero, so the following equation is established [8]:

$N(t)=p \lambda M \zeta$

(9)

(2) When $\lambda_{c}^{\text {loc }}<\lambda<\lambda_{c}$

Some of the nodes in the network are congested, and the average waiting time of the packet is no longer zero, but the whole network is still in stable condition, so the following equation is established:

$N(t)=\frac{p \lambda M \bar{\zeta}}{1-p \lambda M G}$

And because $G=\frac{1}{M^{2}(M-1)^{2} \bar{\zeta}} \sum_{i, j} \sum_{y \in \theta(i, j)} B_{y}$, then $\mathrm{N}(\mathrm{t})$ can be equivalent to that of $N(t)=\frac{p \lambda M \bar{\zeta}}{1-p \lambda\left(1 / P M \lambda_{c}\right)}=p \lambda M \bar{\zeta} \frac{\lambda_{c}}{\lambda_{c}-\lambda}$

(3) When $\lambda>\lambda_{c}$

Network congestion status. According to the evolution rules of the model [9], it can be known that A can be expressed in the following ways:

$N(t)=p \lambda M \frac{\lambda_{c} \bar{\zeta}}{\lambda-\lambda_{c}}\left(e^{\frac{\lambda-\lambda_{c}}{\lambda_{c} \bar{\zeta}}}-1\right)$

\subsubsection{Packet delay behavior analysis}

Now we analyze the variation of the average delay of packets.2.3 Empirical analysis of interest rate risk.

(1) When $\lambda<\lambda_{c}^{\text {loc }}$

The average delay of packets is equal to the mean free delay, i.e., $\bar{\varepsilon}=\bar{\zeta}$.

(2) When $\lambda>\lambda_{c}^{\text {loc }}$

Average delay is also required to add the average waiting time, the average delay of the packet can be expressed as:

$$
\bar{\varepsilon}=\left\{\begin{array}{l}
\bar{\zeta}, \lambda<\lambda_{c}^{l o c} \\
\left(\frac{\lambda_{c}}{\lambda_{c}-\lambda}\right) \bar{\zeta}, \lambda_{c}^{l o c}<\lambda<\lambda_{c} \\
{\left[1+\left(\frac{\lambda_{c}}{\lambda_{c}-\lambda}\right)\left(e^{\frac{\lambda-\lambda_{c}}{\lambda_{c} \zeta}}-1\right)\right] \bar{\zeta}, \lambda>\lambda_{c}}
\end{array}\right.
$$

\subsubsection{The change law of network throughput}

We use $\Omega(t)$ to represent throughput at the time of the network. According to the formula (8), the change law of $\Omega$ can be described as follows:

$$
\frac{d \Omega(t)}{d t}=\frac{N(t)-p M \lambda \bar{\varepsilon}_{w}}{\zeta}
$$


It represents the growth rate of the average throughput of the network. So we can get the following formula (14).

$$
\Omega(t)=\left\{\begin{array}{l}
p M \lambda t ; \lambda<\lambda_{c} \\
p M \lambda_{c}\left(t-\frac{\lambda_{c} \bar{\zeta}}{\lambda-\lambda_{c}} e^{\frac{\lambda_{c} \bar{\zeta}}{\lambda-\lambda_{c}}}\right) ; \lambda>\lambda_{c}
\end{array}\right.
$$

From the above formula we can know that in $\lambda<\lambda_{c}$, the throughput increases linearly with the increase of time. At the $\lambda=\lambda_{c}$ point, the system throughput reaches its maximum limit. When $\lambda>\lambda_{c}$, the throughput decreases exponentially with the increase of time or load, and gradually tends to zero. The above formula shows that when the flow rate is increased to the local phase transition, the local phase transition from stable to unstable occurs in the individual node. At this point, the network has not yet congested nodes have the capacity of handling has not been fully utilized.

\subsection{Numerical simulation}

In this section, we will further verify the theoretical analysis results by numerical simulation. In this section, we will further verify the theoretical analysis results by numerical simulation. For the simulation and analysis of the variation of network performance index under different load conditions, the work in this area has been more abundant in the rule network. In the following simulation experiments, the number of network nodes is taken as 256 . The relative error is defined as $\left.\right|_{x-\hat{x} \mid x}$, where $\mathrm{x}$ is the exact value, $\hat{\mathrm{x}}$ is the simulation value of $\mathrm{x}$.

\section{Empirical PROCESSES}
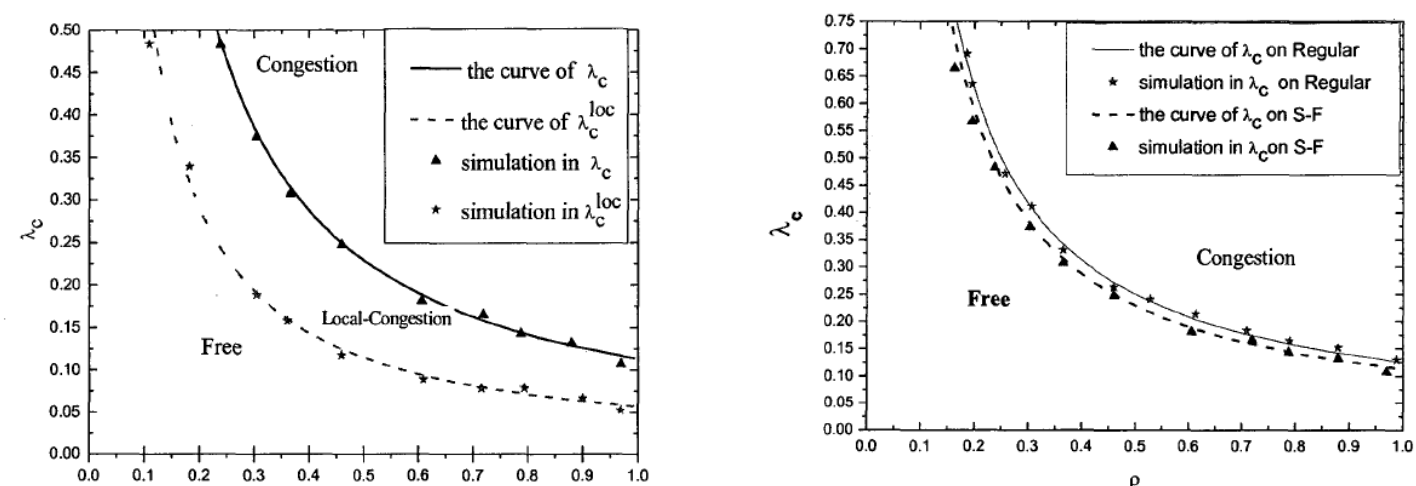

Fig.1 Phase transition traff̂ic vs. p on the free-scale network.

$\lambda_{c}$ between regular and scale-free network
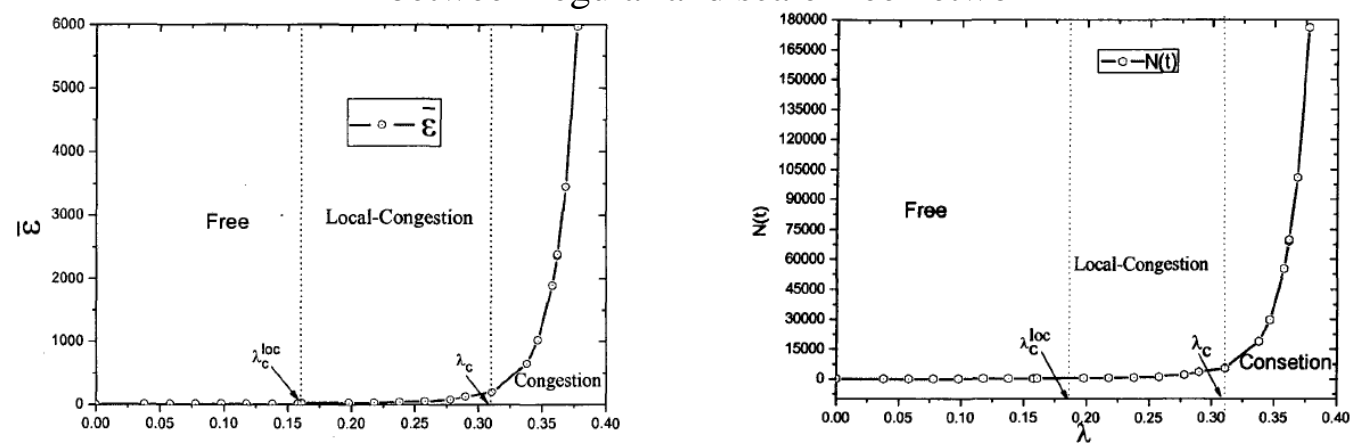

Fig.3 Average delay time of packets vs. $\lambda$ on scale-free network. Fig.4 Number of packets vs. $\lambda$. on scale-free network

Figure 1 indicates the change of phase change flow with $\mathrm{P}$ in the scale free network. The average relative error between the discrete points of $\lambda_{c}^{\text {loc }}$ and the corresponding points of the $\lambda_{c}^{\text {loc }}$ curve is 0.052, and this shows that we have a good estimate of the effect of $\lambda_{c}^{\text {loc }}$ and $\lambda_{c}$ is relatively good. Figure 2 is the simulation results for the estimation of the rule network phase change flow and the comparison of the phase change flow between the regular network and the scale free network. 
Figure 3 and Figure 4 show the variation of queue length, delay, throughput and other performance indicators in the scale free network.

\section{CONCLUSIONS}

By the theoretical analysis and numerical simulation of the related work, we can sum up the following conclusions: (1) the global behavior of heterogeneous network with heterogeneous topology has local characteristics from the free flow regime to the congestion state. That is, the congestion occurs at the first large number of nodes. (2) It is a feasible method to improve the network performance by transforming the network topology to improve the value of the network traffic flow. (3) Homogeneous network local and global phase change flow is approximately equal, which means that the behavior of each node in the uniform network is synchronized or at the same time in a stable state. As the network load increases, the global behavior of the network is directly from the free flow state to the global congestion state. There is no transition state between the two states. (4) When the flow rate exceeds the global phase transition, the queue length and the average delay of the packet are increased dramatically, and the maximum value of the network throughput is quickly reduced to zero.

\section{REFERENCES}

[1] Walton M. Applying complexity theory: A review to inform evaluation design[J]. Evaluation \& Program Planning, 2014, 45(1):119-126.

[2] Landsberg J M. Geometric Complexity Theory: an introduction for geometers[J]. Annali Delluniversità Di Ferrara Sezione Scienze Matematiche, 2015, 61(1):65-117.

[3] Zhou X. Computer Network Behavior Research based on the Theory of Complexity[J]. Applied Mechanics \& Materials, 2014, 556-562(556-562):5822-5824.

[4] He X D, Zhang Y Y. Study on Application of the Complexity Theory in Project management[J]. Applied Mechanics \& Materials, 2014, 584-586:2277-2281.

[5] Acuna J A, Yortsos Y C. Application of fractal geometry to the study of networks of fracture and their pressure transient[J]. Water Resources Research, 1995, 31(3):527-540.

[6] Chua L O, Wang N N. On the application of degree theory to the analysis of resistive nonlinear networks[J]. International Journal of Circuit Theory \& Applications, 1977, 5(1):35-68.

[7] Wu A Y, Zhao Y H, Ma Z G, et al. Graph Theory-Based Multi-Layer State Space Modeling Method of Complex System and Its Application[C]// Proceedings of the 2009 First IEEE International Conference on Information Science and Engineering. IEEE Computer Society, 2009:4895 - 4898.

[8] Zhang S, Li P, Shi R, et al. Study on $\varepsilon$-machine theory method and application in complex system modeling[J]. Chinese Journal of Scientific Instrument, 2014.

[9] Wang F. Modeling a central place system with interurban transport costs and complex rural hinterlands[J]. Regional Science \& Urban Economics, 1999, 29(3):381-409. 